\title{
Progesterone stimulation of fluid absorption by the rat uterine gland
}

\author{
R. J. Naftalin, J. R. Thiagarajah, K. C. Pedley, V. J. Pocock \\ and S. R. Milligan \\ Division of Physiology, King's College London, New Hunt's House, Guys Campus, \\ London SE1 1UL, UK
}

\begin{abstract}
The purpose of this study was to test the hypothesis that the steroid environment affects fluid absorption by the uterine glands. Laser scanning confocal microscopy of the distribution of an extracellular marker (fluorescein isothiocyanate-labelled dextran) within rat uterine glands showed that the endometrial glands change their fluid handling characteristics under different hormonal conditions. Under progesterone dominance, the glands showed an amiloridesensitive dextran accumulation indicating sodium-dependent
\end{abstract}

fluid absorption; however, this was absent in the oestrogendominated state. The rate of fluid uptake in the progesteronestimulated gland opening was estimated to be approximately $1 \times 10^{-4} \mathrm{~cm} \mathrm{~s}^{-1}$, requiring a suction pressure of between 10 and $20 \mathrm{~mm} \mathrm{Hg}$ at the mucosal surface. This study provides the first direct evidence of fluid absorption by the uterine glands. Such absorption may provide the mechanism for closure of the uterine lumen and immobilization of the blastocyst necessary for implantation.

\section{Introduction}

During the ovarian cycle and early pregnancy, the uterus undergoes marked changes, which are controlled by fluctuations in ovarian steroids. Under oestrogen dominance, the uterine lumen of rats and mice 'balloons' out due to fluid secretions, but most of this fluid is lost through the cervix after ovulation (Armstrong, 1968; Kennedy and Armstrong, 1975). However, the uterus maintains an obvious fluid-filled lumen until near the time of implantation, but even this fluid eventually disappears in the peri-implantation period. This complete 'closure' of the uterus is a characteristic feature of many species that have small blastocysts (for example, rats, mice and hamsters) (Martin et al., 1970; Hedlund et al., 1971, 1972; Ljungkvist, 1972) and provides a mechanism by which embryos are held in contact with the uterine epithelium before initiation of implantation. It must be emphasized that this uterine closure is very tight: the microvilli on the opposing epithelia become tightly interdigitated, resulting in the complete obliteration of the lumen as observed under an electron microscope (Martin et al., 1970). The few observations that have been made in humans and primates indicate that there is a similar reduction or loss of the uterine luminal volume. Casslen (1986) estimated the volume of human luminal fluid to be only 5-35 $\mu$ in the luteal phase of the oestrous cycle, which is consistent with the observations of Maier and Kuslis (1988).

Email: richard.naftalin@kcl.ac.uk
The changes in the volume of uterine fluid reflect net fluid movement across both the luminal and glandular epithelial surfaces. Secretion is generally ascribed to the glands and absorption is attributed to the luminal epithelium (Clemetson et al., 1977; Beier and Beier-Hellwig, 1998). There is electrogenic $\mathrm{NaCl}$ absorption across mouse endometrial epithelium that can be stimulated by prostaglandins opening $\mathrm{K}^{+}$channels in the epithelial basolateral membranes (Wang and Chan, 2000). Endometrial electrolyte absorption in mice is inhibited by amiloride, an inhibitor of apical membrane $\mathrm{Na}^{+}$conductance channels (Wang and Chan, 2000). Isolated epithelial cells in the human endometrium also show an amiloride-sensitive sodium conductance that may be linked to fluid transport (Matthews et al., 1998). In addition to the above mechanisms, the simultaneous appearance of luminal epithelial cell endocytosis and uterine closure in rats led to the suggestion that the uterine luminal fluid may be removed by the endocytotic activity of the epithelial cells (Parr, 1983). Characteristic irregular cytoplasmic projections ('pinopodes') appear on the luminal surface of epithelial cells in humans and rats near the time of uterine receptivity and may play a role in fluid absorption (Enders and Nelson, 1973; Parr and Parr, 1974; Leroy et al., 1976).

The purpose of the present study was to test the hypothesis that uterine glands could play a major role in the process of uterine fluid absorption, analogous to the role of crypts in absorption of water by the colon (Naftalin and Pedley, 1999). Uterine glands are usually only considered to be secretory and are known to play a role in producing an appropriate environment for sperm transport and for the early embryo. The possibility that the glands switch from 
secretion to absorption would provide a mechanism for reducing luminal fluid volume and could support, or even drive, the closure of the uterine lumen. Additional experiments were undertaken to investigate this hypothesis using laser scanning confocal microscopy of the distribution of the extracellular marker fluorescein isothiocyanate-labelled dextran (FITC-dextran; molecular mass $10 \mathrm{kDa}$ ) in the luminae of glands of isolated rat uteri under either oestrogen or progesterone domination.

\section{Materials and Methods}

\section{Tissue preparation}

Intact adult female Wistar rats weighing 200-250 g were pretreated for 3 days with either oestradiol $(10 \mu \mathrm{g}$ per animal per day, s.c. injection in arachis oil) or progesterone (5 mg per animal per day, s.c. injection in arachis oil, $n=4$ rats per treatment). A second series of experiments was undertaken to examine the effects of amiloride on progesterone-treated rats $(n=3)$. On day 3 , the uteri from freshly killed rats were gently inflated by an injection with $<0.3 \mathrm{ml}$ Earle's-Hepes (pH 7.35, containing $124.0 \mathrm{mmol}$ $\mathrm{NaCl} \mathrm{I}^{-1}, 5.4 \mathrm{mmol} \mathrm{KCl} \mathrm{I-1}^{-1} 0.8 \mathrm{mmol} \mathrm{MgSO}_{4} \mathrm{I}^{-1}, 1.0 \mathrm{mmol}$ $\mathrm{NaHPO}_{4} \mathrm{I}^{-1}, 14.3 \mathrm{mmol} \mathrm{NaHCO}{ }_{3}{ }^{-1}, 10.0 \mathrm{mmol}$ Hepes ${ }^{-1}$, $1.8 \mathrm{mmol} \mathrm{CaCl}_{2} \mathrm{~L}^{-1}, 5.5 \mathrm{mmol}$ glucose $\mathrm{I}^{-1}$ ), isolated and opened along their anti-mesometrial border. Dissected full thickness mucosa with an intact supporting myometrial layer was mounted as a $2-3 \mathrm{~mm}^{2}$ sheet in a temperaturecontrolled perfusion chamber at $37^{\circ} \mathrm{C}$. FITC-dextran (10 kDa; $20 \mu \mathrm{mol} \mathrm{I}^{-1}$ in Earle's-Hepes) was introduced to the perfusion chamber and allowed to accumulate within the glands. Amiloride $\left(0.1 \mathrm{mmol} \mathrm{I}^{-1}\right)$ in FITC-dextran solution was added to tissue from progesterone-treated animals after $20 \mathrm{~min}$ in normal FITC-dextran solution.

\section{Confocal microscopy}

Laser scanning confocal microscopy was used to investigate the distribution of the extracellular marker (FITC-dextran) in the gland luminae of isolated rat uteri. The tissue was viewed using a Nikon Diaphot inverted microscope with a Nikon Fluor $\times 20$ or $\times 60$ Plan Achromat lens. The microscope was attached to an MRC 600 confocal scanhead equipped with two detection channels and an $\mathrm{Ar}-\mathrm{Kr}$ mixed gas laser allowing excitation at 488 and $568 \mathrm{~nm}$. A software-controlled stepper motor attached to the fine focus control provided $z$-axis movement with $0.1 \mu \mathrm{m}$ resolution. Each piece of tissue was viewed using the confocal microscope from the mucosal side (face down) and two to four glands were examined in each piece. The focus plane was initially taken to the surface of the tissue and images were captured at intervals of $5 \mu \mathrm{m}$ using the automatic Z-step motor from 0 to $40 \mu \mathrm{m}$ below the surface. The captured images were analysed using the public domain NIH Image program (written by W. Rasband at the US National Institute of Health) to quantify the fluorescence. Fluorescence was evaluated by measuring the mean fluorescence intensity of a designated region at each of the depths observed. This procedure was repeated for all time points.

\section{Estimation of the average concentration of FITC-dextran in the lumen of uterine glands}

The luminal concentration of FITC-dextran in the uterine gland was estimated by monitoring the ratio of fluorescence intensity of the dye in the lumen of the gland at any depth, $x$, to that in the luminal opening of the gland, $x=0$. The relative changes in luminal concentrations were quantified using fluorescence after attenuation of the signal with a neutral density filter (3\% transmittance) and subtraction of background. With this high level of neutral density filtration, there is no detectable autofluorescence from the tissue.

The ratio $\left(I_{x}-I_{\mathrm{bkg}}\right):\left(I_{\mathrm{go}}-l_{\mathrm{bkg}}\right)$ in the lumen of the gland is equivalent to the concentration polarization at varied depths, $x$, (where $I_{x}$ is the fluorescence intensity in the lumen of the gland at distance $x$ from the luminal opening of the gland, $l_{\mathrm{go}} ;$ and $l_{\mathrm{bkg}}$ is the fluorescence in the absence of dye; $l_{\text {bkg }}=30-50$ units per pixel). Estimates of average fluorescence intensity in the lumen of the gland were obtained by taking the pixel intensity of circumscribed areas of interest within 15-18 lumens per point, 500-1000 pixels per area. The maximum pixel light intensity $=256$ units -background. The intensities of the same regions in the gland lumen at successive depths at progressive increases in time were computed.

\section{Fluorescence staining of F-actin with Alexa 488- phalloidin}

Isolated endometrial mucosa (progesterone-treated) was fixed for $30 \mathrm{~min}$ at $37^{\circ} \mathrm{C}$ in $2 \%(\mathrm{v} / \mathrm{v})$ paraformaldehyde in PBS and then permeabilized for $30 \mathrm{~min}$ in $0.2 \%(\mathrm{v} / \mathrm{v})$ Triton-X100 in PBS at room temperature. The permeabilized mucosa was then bathed in PBS buffer containing a specific stain for F-actin, Alexa 488 phalloidin (Molecular Probes, Eugene, OR) 1:100 for 30 min. Excess stain was removed by repeated washing in buffer.

\section{Statistical analysis}

Results were analysed using the Student's $t$ test (unpaired, two-tailed, with Bonferroni correction for multiple comparisons) and $P<0.05$ was considered significant. All results are expressed as means \pm SEM.

\section{Results}

\section{Confocal microscopy of fluorescence distribution of FITC-dextran in uterine glands}

Optical sections along the uterine gland axis showed that the fluorescence distribution of FITC-dextran reached a quasi steady state after 20 min exposure. The changes in fluorescence intensities with increasing depth along a single representative gland lumen from the uterus of either an oestradiol- or progesterone-treated animal are shown (Fig. 1). 
(a)

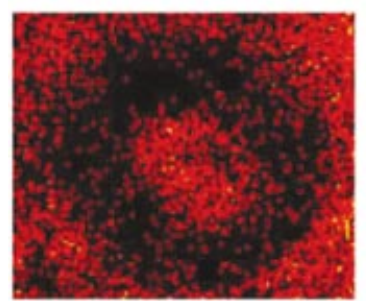

$5 \mu \mathrm{m}$

(b)

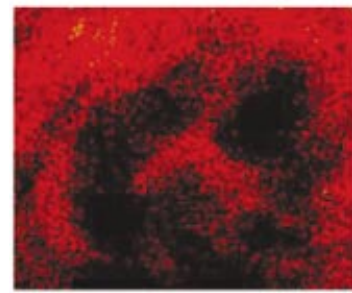

$5 \mu \mathrm{m}$

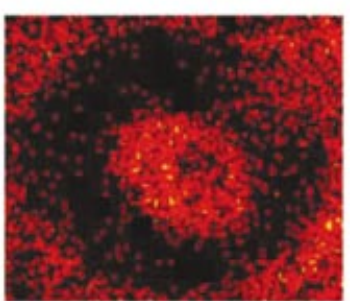

$10 \mu \mathrm{m}$

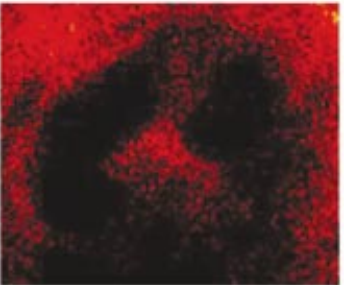

$10 \mu \mathrm{m}$

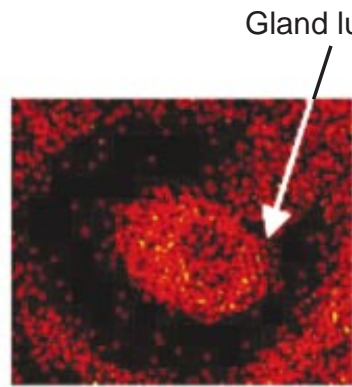

$15 \mu \mathrm{m}$

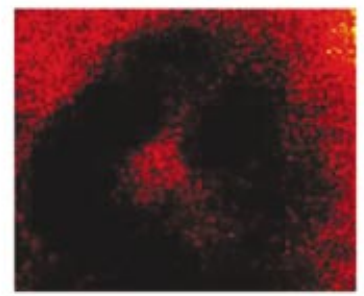

$15 \mu \mathrm{m}$

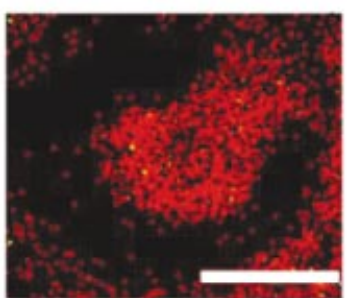

$20 \mu \mathrm{m}$

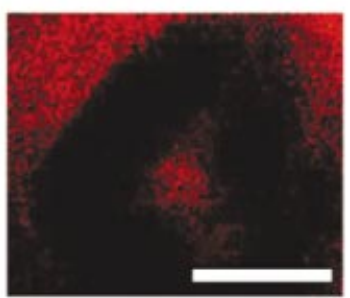

$20 \mu \mathrm{m}$

Fig. 1. Confocal images of endometrial glands showing fluorescein isothiocyanate (FITC)-dextran accumulation and F-actin distribution. (a) Glands from progesterone-treated rats showing increasing dextran accumulation with increasing depth into the gland lumen. (b) Glands from oestrogen-treated animals showing the decrease in dextran accumulation in gland lumen with increasing depth into the gland. Scale bars represent $50 \mu \mathrm{m}$.

The normalized fluorescence intensities in glands from animals treated with either oestradiol or progesterone are shown (Fig. 2a). It is evident that there is increased FITC-dextran accumulation in the proximal part of progesterone-treated glands. This pattern of accumulation of the extracellular marker is consistent with convectiondriven entry of dye into the lumina of the glands, followed by fluid absorption through the gland walls (Naftalin and Pedley, 1999). Similar patterns of accumulation of FITC-dextran have been observed in colonic crypts (Thiagarajah et al., 2000). In tissue from oestradiol-treated animals, there was a continuous decrease in FITC-dextran concentration along the length of the gland, which was consistent with simple diffusive entry of dye into the gland lumen.

\section{Estimation of the average concentration of FITC-dextran in the gland lumen}

Simulation of the coupled fluxes of dextran, fluid and electrolyte using a computer program originally designed to model movements in colonic crypts showed that the rate of dextran accumulation in the lumen of a progesteronetreated uterine gland was $1 \times 10^{-4} \mathrm{~cm} \mathrm{~s}^{-1}$. This finding is consistent with the observed $t_{1 / 2}=12.6 \pm 4.4 \mathrm{~min}$ (Fig. 2b). This value is only about $5 \%$ of the rate found in colonic crypts, implying that the suction tension generated by the endometrial glands is much lower than that of the colon. The osmotic gradient needed to generate this flow is only 1-5 mOsm kg-1. This gradient would generate a suction tension at the gland opening of $10-20 \mathrm{~cm} \mathrm{H}_{2} \mathrm{O}$.

Amiloride $\left(0.1 \mathrm{mmol} \mathrm{I}^{-1}\right)$ treatment of the uterus from progesterone-treated animals quickly inhibited accumulation of FITC-dextran in the gland lumen (Fig. 3a). Before the application of amiloride, the FITC concentration at 5-15 $\mu \mathrm{m}$ within the glands was significantly higher than at the gland opening $(P<0.001)$. After 4 min of amiloride application, there was a complete attenuation of the FITC accumulation $(P<0.001)$. The time course at a single depth (Fig. 3b) shows that the ratio of FITC fluorescence (relative to that at the opening) rapidly diminished (Student's $t$ test; $P<0.001$ ). This finding is consistent with fluid absorption being dependent on active $\mathrm{Na}^{+}$transport across the glandular epithelium.

Observations in the present study revealed that endometrial glands open at the bottom of folds in the surface mucosal layer. These folds project 5-10 $\mu \mathrm{m}$ above the gland opening. This arrangement differs from colonic crypts in rats and humans, which open directly on to the surface lumen. The F-actin staining (Fig. 4) shows the basic structure and dimensions of the larger endometrial glands (approximately 5000 large glands $\mathrm{cm}^{-2}$ of endometrial surface). 
(a)

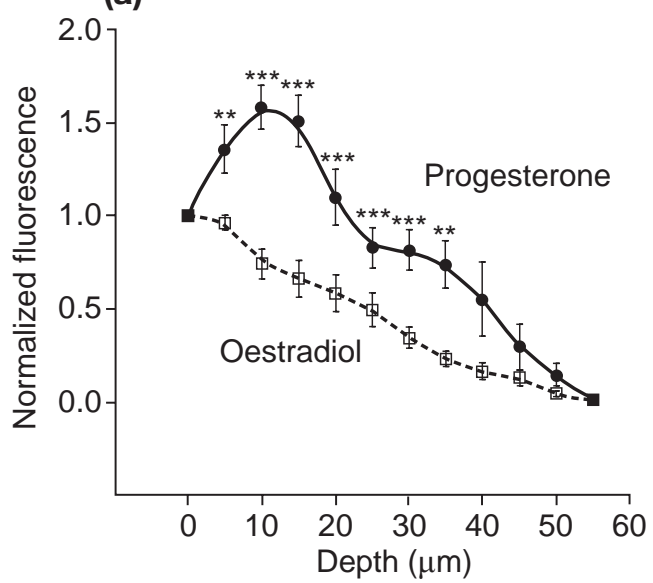

(b)

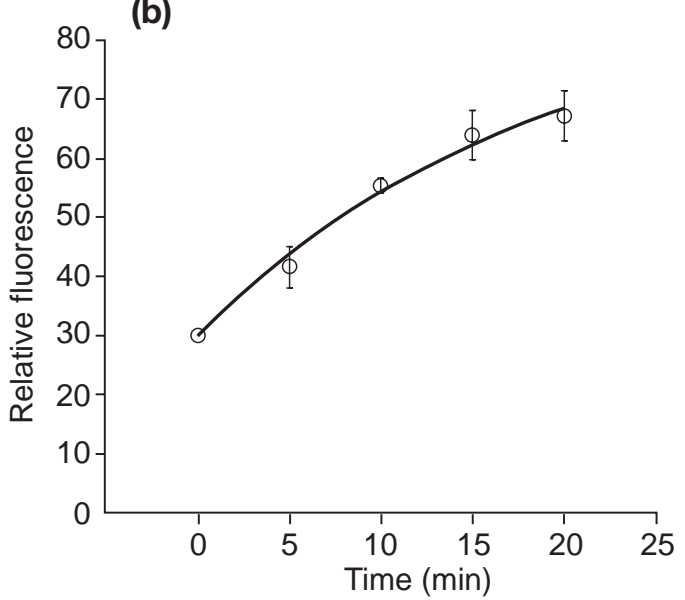

Fig. 2. (a) Comparison of steady-state dextran accumulation in glands from progesterone- $(\mathbf{)}$ ) and oestrogen( $\square$ ) treated rats after 20-30 min. Data normalized to the values at the gland opening $=1$ and at a basal level at $55 \mu \mathrm{m}$, where the signal to noise ratio $=1$ (each point represents the means of \pm SEM of 12 glands from a total of four rats per treatment). There is a significant increase in dextran accumulation at depths between 5 and $40 \mu \mathrm{m}$ in progesterone- versus oestrogen-treated glands. The attenuation of the fluorescence signal with increasing depth within the tissue is mainly due to the absorption and scatter of both excitation and emitted light by overlying tissue. Consequently, the accumulation ratio is likely to be a more realistic representation of the dye accumulation with depth in progesterone-treated tissue ${ }^{* *} P<0.01,{ }^{* * *} P<0.001 ; t$ test with Bonferroni correction for multiple comparisons). (b) Time course of fluorescein isothiocyanate (FITC)-dextran uptake into progesterone-treated rat endometrial glands at a depth of $30 \mu \mathrm{m}$ below the surface. The time course is fitted to a mono-exponential $t_{1 / 2}=12.6 \pm 4.4 \mathrm{~min}$.

(a)

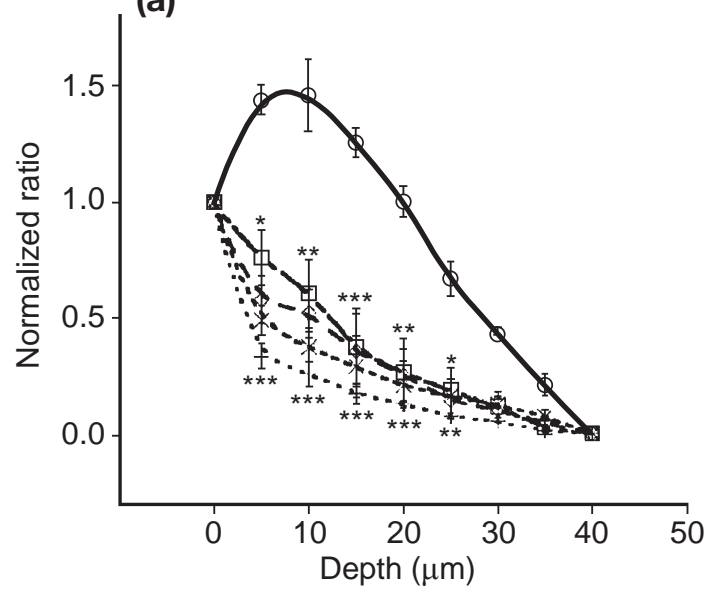

(b)

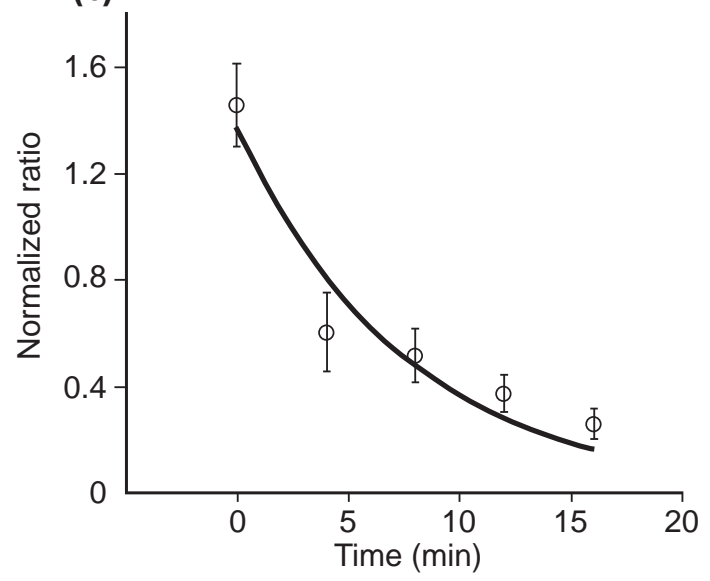

Fig. 3. (a) Effect of amiloride $\left(0.1 \mathrm{mmol}^{-1}\right)$ on the time course $(0 \mathrm{~min}, 0 ; 4 \mathrm{~min}, \square ; 8 \mathrm{~min}, \diamond ; 12 \mathrm{~min}, \times ; 16 \mathrm{~min},+)$ and distribution of fluorescein isothiocyanate (FITC)-dextran in uterine glands from progesterone-treated rats. There is a significant decrease in FITC-dextran accumulation at depths between 5 and $30 \mu \mathrm{m}(P<0.001 ; n=3$ rats, nine glands). There is also a change in the pattern of the dextran distribution between 0 and 4 min: before amiloride exposure, dextran concentration is higher at depths between 5 and $15 \mu \mathrm{m}$ than at the gland opening whereas after amiloride treatment, dextran concentration is lower at all depths than at the gland opening $\left(* P<0.025,{ }^{* *} P<0.01\right.$, ***$P<0.001 ; t$ test with Bonferroni correction applied for multiple comparisons; upper and lower asterisks refer to 4 and $16 \mathrm{~min}$, respectively, after application of amiloride). (b) Time course of effect of amiloride $\left(0.1 \mathrm{mmol}^{-1}\right) 15 \mu \mathrm{m}$ below the gland opening. The $y$ axis shows the ratio of dextran accumulation at $15 \mu \mathrm{m}$ to that at the gland opening. The rate of decrease of the dextran accumulation has a $t_{1 / 2}$ of $5.3 \pm 1.1 \mathrm{~min}(P<0.001)$. 


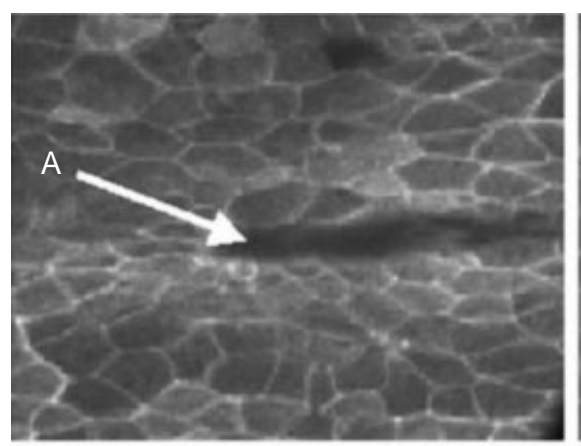

$0 \mu \mathrm{m}$

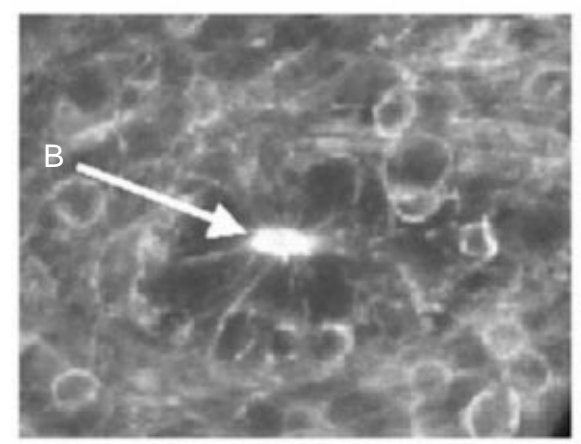

$15 \mu \mathrm{m}$

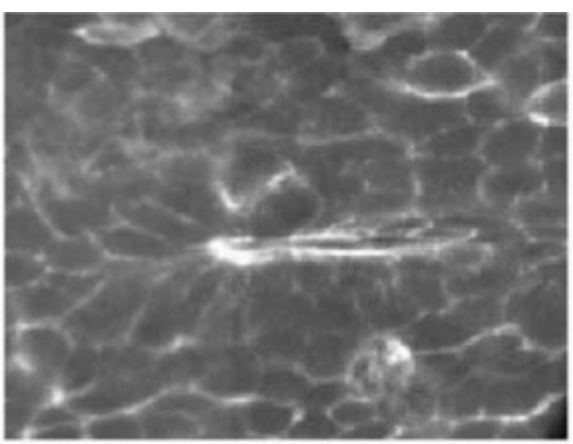

$5 \mu \mathrm{m}$

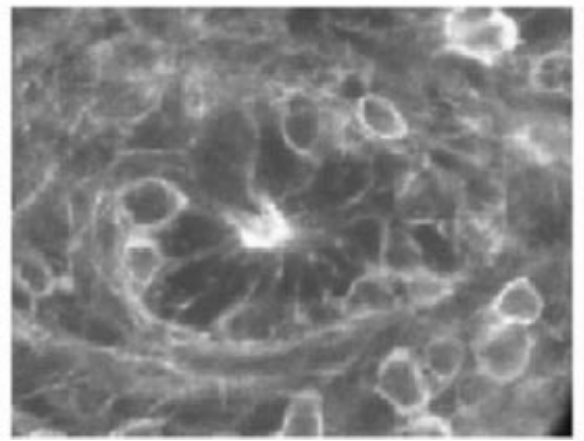

$20 \mu \mathrm{m}$

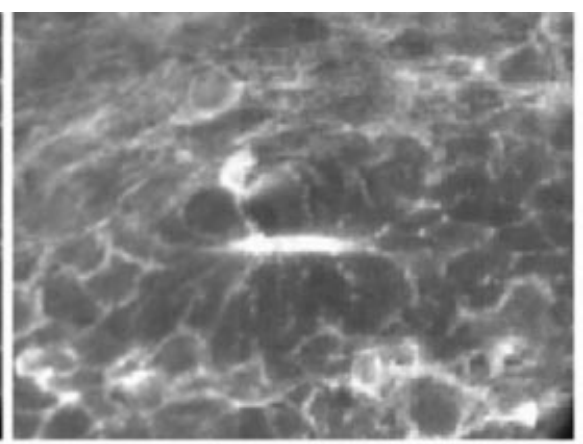

$10 \mu \mathrm{m}$

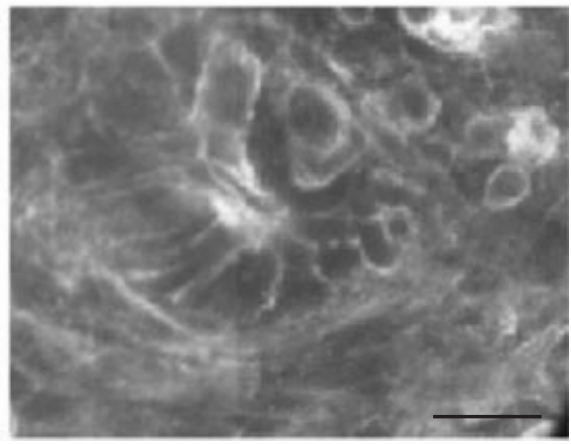

$25 \mu \mathrm{m}$

Fig. 4. Confocal images of Alexa-488 phalloidin stain for F-actin in rat uterine glands with depth, showing the bright staining brush border microvilli and structure of large glands. The gland openings (B) open at the bottom of mucosal folds (A), approximately $15 \mu \mathrm{m}$ below the endometrial surface. Scale bar represents $40 \mu \mathrm{m}$.

\section{Discussion}

The present study provides the first direct evidence that endometrial glands have an absorptive function that is stimulated under conditions of progesterone dominance. These studies were undertaken in intact animals treated with a chronic steroid regimen designed to establish either oestrogen- or progesterone-dominance without any prior surgical interference. Further elucidation of the hormone control of fluid handling will require studies on ovariectomized, steroid-treated animals to determine the time course of effects and specific interactions between the hormones.

By applying a suction force at the endometrial surface, uterine glands would contribute to the uterine closure in the peri-implantation period which establishes the first close embryo-maternal contact by immobilizing the blastocyst between opposing luminal epithelial walls (Fig. 5). This possible role for uterine glands in the closure reaction has been overlooked previously, presumably reflecting the general attribution of a secretory, rather than an absorptive, activity to glands.

Endometrial glands have similarities to colonic crypts: both are blind-ended mucosal tubes surrounding a lumen of 5-20 $\mu \mathrm{m}$ and both mucosae express amiloride-sensitive epithelial $\mathrm{Na}^{+}$conductance channels (Bridges et al., 1989; Naftalin and Pedley, 1999; Chan et al., 2000). A major difference is the absence of any peri-glandular sheath surrounding the endometrial glands: in the colon the myofibroblastic sheath acts as an effective barrier to $\mathrm{Na}^{+}$ movement and permits build up of a large osmotic gradient (2-5 atmospheres) across the crypt wall (Thiagarajah et al., 2001a). The absence of a peri-glandular structure in the endometrium means that there can be no large-scale electrolyte accumulation adjacent to the basement membranes of the gland epithelium. The maximum accumulation of FITC-dextran observed within the progesterone-treated glands was only 1.5 times higher than that in the bathing solution and dextran accumulation in glands was relatively slow $\left(t_{1 / 2}=12.6 \pm 4.4 \mathrm{~min}\right)$ compared with that of murine colonic crypts $\left(t_{1 / 2}\right.$ of $1-2 \mathrm{~min}$ in vitro; $0.3 \mathrm{~min}$ in vivo (Thiagarajah et al., 2001a,b). Additional evidence for a lack of any peri-glandular sequestration of ions is the rapidity of action of amiloride $\left(t_{1 / 2}=5.3 \pm 1 \mathrm{~min}\right)$ on dextran accumulation in the glands from progesterone-treated animals. In contrast, amiloride treatment in murine colonic crypts reduces accumulation much more slowly $\left(t_{1 / 2}=\right.$ $41.7 \pm 3.4 \mathrm{~min}$ ) because of the sequestration of $\mathrm{Na}^{+}$in the peri-cryptal sheath (Thiagarajah et al., 2001a).

The mechanisms controlling the absorptive process within the glands and the relative contribution of the glandular and luminal epithelial surfaces in luminal fluid absorption remain to be investigated. The basal transport properties of isolated pig endometrial epithelial cells are regulated by a number of factors, including prostaglandins, epidermal growth factor (EGF), transforming growth factor $\alpha$ 


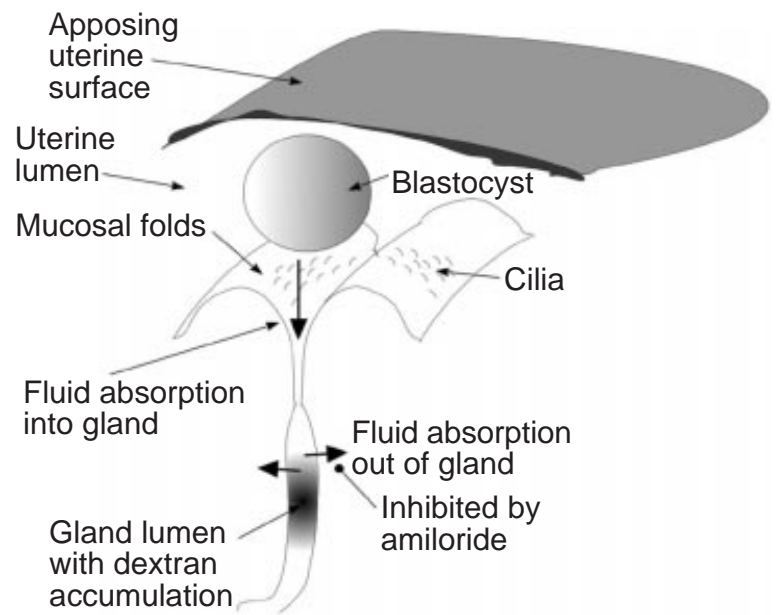

Fig. 5. Representation showing how fluid suction into endometrial glands of rats would result in uterine closure and immobilization of the blastocyst between the mucosal folds.

(TGF $\alpha$ ) and insulin (Deachapunya and O'Grady, 1998, 2001), and the ratio of progesterone:oestradiol regulates the expression of the amiloride-sensitive epithelial $\mathrm{Na}^{+}$ conductance channels in rat lung (Sweezey et al., 1998). Progesterone also regulates expression of factors, such as $\beta$ catenin and cadherin-11, in the endometrium (Chen et al., 1998). These observations indicate that progesterone may have a key role in regulating the adhesion between glandular epithelial cells and the underlying stroma as well as activating the epithelial $\mathrm{Na}^{+}$transport process upon which fluid transport depends. If the glands do contribute significantly to uterine closure, any disturbances of the absorptive mechanism (such as those induced by inappropriate steroid exposure) would be expected to disrupt closure and implantation. These data were communicated at the York meeting of the Physiological Society 2001 (Naftalin et al., 2002).

R. J. Naftalin and K. C. Pedley are grateful to the Wellcome Trust for support of this work.

\section{References}

Armstrong DT (1968) Hormonal control of uterine lumen fluid retention in the rat American Journal of Physiology 214 764-771

Beier HM and Beier-Hellwig K (1998) Molecular and cellular aspects of endometrial receptivity Human Reproduction Update 4 448-458

Bridges RJ, Cragoe EJ, Frizzell RA and Benos DJ (1989) Inhibition of colonic $\mathrm{Na}^{+}$transport by amiloride analogs American Journal of Physiology 256 C67-C74

Casslen B (1986) Uterine fluid volume. Cyclic variations and possible extra uterine contributions Journal of Reproductive Medicine 31 506-510

Chan LN, Wang XF, Tsang LL, Liu CQ and Chan HC (2000) Suppression of CFTR-mediated $\mathrm{Cl}(-)$ secretion by enhanced expression of epithelial $\mathrm{Na}\left({ }^{+}\right)$channels in mouse endometrial epithelium Biochemical and Biophysical Research Communications 276 40-44

Chen GTC, Getsios S and MacCalman CD (1998) Progesterone regulates beta-catenin mRNA levels in human endometrial stromal cells in vitro. Endocrine 9 263-267

Clemetson CA, Verma UL and De Carlo SJ (1977) Secretion and reabsorption of uterine luminal fluid in rats Journal of Reproduction and Fertility 49 183-187
Deachapunya C and O'Grady SM (1998) Regulation of chloride secretion across porcine endometrial epithelial cells by prostaglandin E2 Journal of Physiology 508 31-47

Deachapunya C and O'Grady S (2001) Epidermal growth factor regulates the transition from basal sodium absorption to anion secretion in cultured endometrial epithelial cells Journal of Cell Physiology $\mathbf{1 8 6} 243-250$

Enders AC and Nelson DM (1973) Pinocytotic activity of the uterus of the rat American Journal of Anatomy 138 277-300

Hedlund K and Nilsson $\mathbf{O}$ (1971) Hormonal requirements for the attachment reaction and blastocyst implantation in the mouse, hamster and guinea-pig Journal of Reproduction and Fertility 26 267-269

Hedlund K, Nilsson O, Reinus S and Aman G (1972) Attachment reaction of the uterine luminal epithelium at implantation: light and electron microscopy of the hamster, guinea-pig, rabbit and mink Journal of Reproduction and Fertility 29 131-132

Kennedy TG and Armstrong DT (1975) Loss of uterine luminal fluid in the rat: relative importance of changing peripheral levels of estrogen and progesterone Endocrinology 97 1379-1385

Leroy F, van Hoeck J and Bogaert C (1976) Hormonal control of pinocytosis in the uterine epithelium of the rat Journal of Reproduction and Fertility 47 59-62

Ljungkvist I (1972) Attachment reaction of rat uterine luminal epithelium. IV. The cellular changes in the attachment reaction and its hormonal regulation Fertility and Sterility 23 847-865

Maier DB and Kuslis ST (1988) Human uterine luminal fluid volumes and prolactin levels in normal menstrual cycles American Journal of Obstetrics and Gynecology 159 434-439

Martin L, Finn CA and Carter J (1970) Effects of progesterone and oestradiol on the luminal epithelium of the mouse uterus Journal of Reproduction and Fertility 21 461-469

Matthews CJ, McEwan GT, Redfern CP, Thomas EJ and Hirst BH (1998) Absorptive apical amiloride-sensitive $\mathrm{Na}^{+}$conductance in human endometrial epithelium Journal of Physiology 513 443-452

Naftalin RJ and Pedley KC (1999) Regional crypt function in rat large intestine in relation to fluid absorption and growth of the pericryptal sheath Journal of Physiology (London) 514 211-227

Naftalin RJ, Thiagarajah JR, Pedley KC, Pocock VJ and Milligan SR (2002) Progesterone stimulation of fluid absorption by the rat uterine gland Journal of Physiology 539.P, 7P (research communication)

Parr MB (1983) Relationship of uterine closure to ovarian hormones and endocytosis in the rat Journal of Reproduction and Fertility 68 185-188

Parr MB and Parr EL (1974) Uterine luminal epithelium: protrusions mediate endocytosis, not apocrine secretion, in the rat Biology of Reproduction 11 220-233

Sweezey N, Tchepichev S, Gagnon S, Fertuck K and O'Brodovich H (1998) Female gender hormones regulate mRNA levels and function of the rat lung epithelial Na channel American Journal of Physiology (Cell Physiology) 274 379-386

Thiagarajah JR, Gourmelon P, Griffiths NM, Lebrun F, Naftalin RJ and Pedley KC (2000) Radiation induced cytochrome c release causes loss of rat colonic fluid absorption by damage to crypts and pericryptal myofibroblasts Gut 47 675-684

Thiagarajah JR, Jayaraman S, Naftalin RJ and Verkman AS (2001a) In vivo fluorescence measurement of $\mathrm{Na}^{+}$concentration in the pericryptal space of mouse descending colon American Journal of Physiology and Cell Physiology 281 C1898-C1903

Thiagarajah JR, Pedley KC and Naftalin RJ (2001b) Evidence of amiloride sensitive fluid absorption in rat descending colonic crypts from confocal microscopic studies of fluorescence recovery of FITC-labelled dextrans after photobleaching Journal of Physiology 536 541-553

Wang XF and Chan HC (2000) Adenosine triphosphate induces inhibition of $\mathrm{Na}^{+}$absorption in mouse endometrial epithelium: a Ca ${ }^{2+}$-dependent mechanism Biology of Reproduction 63 1918-1924

Received 20 November 2001.

First decision 11 January 2002.

Revised manuscript received 17 January 2002.

Accepted 17 January 2002. 